\title{
Metabolic risks, white matter hyperintensities, and arterial stiffness in high-functioning healthy adults
}

\author{
Hsu-Ko Kuo a,b,c,1, Ching-Yu Chen ${ }^{\text {b, } 1}$, Hon-Man Liu ${ }^{\text {d,1 }}$, Chung-Jen Yen ${ }^{\text {c }}$, King-Jen Chang ${ }^{\text {e }}$, \\ Chien-Cheng Chang ${ }^{\mathrm{f}}$, Yau-Hua Yu ${ }^{\mathrm{g}}$, Lian-Yu Lin ${ }^{\mathrm{c}, *}$, Juey-Jen Hwang ${ }^{\mathrm{c}, \mathrm{h}, *}$ \\ a Department of Geriatrics and Gerontology, National Taiwan University Hospital, Taipei, Taiwan \\ ${ }^{\mathrm{b}}$ Division of Gerontology Research, National Health Research Institutes, Taipei, Taiwan \\ ${ }^{\mathrm{c}}$ Department of Internal Medicine, National Taiwan University Hospital, Taipei, Taiwan \\ d Department of Medical Imaging, National Taiwan University Hospital, Taipei, Taiwan \\ ${ }^{\mathrm{e}}$ Department of Surgery, National Taiwan University Hospital, Taipei, Taiwan \\ ${ }^{\mathrm{f}}$ Division of Mechanics, Research Center for Applied Sciences, Academia Sinica, and Institutes of Applied Mechanics, National Taiwan University, Taipei, Taiwan \\ ${ }^{g}$ School of Dentistry, National Yang-Ming University and Department of Dentistry and the Department of Medical Research and Education, Veterans General Hospital, \\ Taipei, Taiwan \\ ${ }^{\mathrm{h}}$ College of Medicine, National Taiwan University, Taipei and Cardiovascular Center, National Taiwan University Hospital Yun-Lin Branch, Douliou City, Taiwan
}

\begin{abstract}
Background: Arterial stiffness, an age-related feature and measured noninvasively by pulse wave velocity (PWV), is associated with a variety of cardiovascular diseases. Although white matter lesion (WML), representing cerebrovascular micro-angiopathy, is typically considered as a preclinical cardiovascular disease, little is know about the association between PWV and WML. The aim of this study is to examine the association between PWV and WML.

Method: We examined the extent of white matter lesion on cranial magnetic resonance imaging of 93 participants (mean 72.46 years) from the annual Health Examination for the Elderly Program in the National Taiwan University Hospital. Two subtypes of WML including periventricular hyperintensity $(\mathrm{PVH})$ and deep white matter hyperintensity (DWMH) were graded according to Fazekas et al. The brachialankle PWV (baPWV) and heart-ankle PWV (haPWV) were measured using an automatic waveform analyzer.

Results: PVH and DWHM were found in $71(76 \%)$ and $58(62 \%)$ participants. Various degree of WML was observed in $80(86 \%)$ participants. Hypertension, use of anti-hypertensive medications, and elevated blood pressure were identified as risk factors of WML. The baPWV and haPWV were correlated with many metabolic risks, including systolic blood pressure, pulse pressure, and serum triglycerides. The baPWV and haPWV were greater in participants with higher grades of WML in the multivariate analyses. The receiver operating characteristics analyses demonstrated that area under curves for haPWV to identify moderate-to-severe PVH and DWMH were 0.78 (95\% confidence interval $[\mathrm{CI}] 0.66-0.91)$ and 0.72 (95\% CI 0.59-0.85), respectively.

Conclusion: Greater PWV is associated with higher grades of WML among high-functioning older adults. Our findings support the notion that measurement of arterial stiffness is useful in clinical practice for detection of preclinical cerebrovascular disease.
\end{abstract}

(C) 2009 Published by Elsevier Ireland Ltd.

Keywords: Arterial stiffness; Pulse wave velocity; White matter lesions; Leukoaraiosis

\footnotetext{
* Corresponding authors. Hwang is to be contacted at National Taiwan University Hospital Yun-Lin Branch, 579 Yun-Lin Road, Sec 2, Dou-Liou City, YunLin County 640, Taiwan. Lin, Department of Internal Medicine, National Taiwan University Hospital, 5 Chung-Shan South Road, Taipei 100, Taiwan.

E-mail addresses: lin7010@ms1.hinet.net (L.-Y. Lin), jueyhwang@ntu.edu.tw (J.-J. Hwang).

${ }^{1}$ These authors have equal contribution to this paper.
} 


\section{Introduction}

Magnetic resonance imaging (MRI) of the brain in elderly people commonly shows incidental T2-weighted hyperintensities in the periventricular or subcortical areas, often reported as "white matter lesion" (WML). Recent epidemiological studies have reported a high prevalence of WML in the aging brains. Cardiovascular Health Study, a large population-based cohort study, performed MRIs on community-dwelling older adults from 4 US communities, and found that WML was present in $87 \%$ of all participants [1]. The Rotterdam Scan Study of the Netherlands reported a even higher prevalence of WML, with only $5 \%$ of community-dwelling older adults had no WML in either periventricular or subcortical white matter [2]. WML, although often overlooked, has important clinical implication because of its close association with multi-system agerelated problems, including falls, gait problems, cognitive impairment, depression, and urinary incontinence [3]. It is generally believed that the pathogenesis of WML, although remained largely unknown, involves chronic cerebral hypoperfusion [4], cerebral ischemia [5], as well as atherosclerosis[6]. Advanced age, hypertension [7,8], diabetes mellitus [9], hemodynamic dysregulations [10,11], as well as such genetic factors as Notch3 gene [12] and angiotensin-converting enzyme gene [13] have been identified as important risk factors for WML.

Arterial stiffening is a common feature of aging and is accelerated by cardiovascular risk factors and disorders such as hypertension $[14,15]$, diabetes $[15,16]$ and renal failure [17]. Pulse wave velocity (PWV), a direct measure of arterial stiffness, is an independent predictor of many cardiovascular diseases such as stroke and ischemic heart disease [18]. WML, representing cerebrovascular micro-angiopathy, is typically considered as a "preclinical" cardiovascular disease. Thus, the aim of this study is to test the hypothesis that arterial stiffness - as measured by PWV - is associated with subclinical cerebrovascular microangiopathy, namely, WML. In addition, the usefulness of PWV as a surrogate marker in identifying radiographically evident WML is to be examined. We seek to achieve the study goals by analyzing data in a group of high-functioning older adults who participated in the Health Examination for the Elderly Program (HEEP) in the National Taiwan University Hospital (NTUH), a tertiary medical center in Taipei, Taiwan.

\section{Methods}

\subsection{Source of study participants}

The HEEP, a health service funded by Taipei City Government, was opened to senior citizens of Taipei City who are aged 65 or older. The HEEP is provided in 6 tertiary medical centers and 23 community hospitals across Taipei city. The Kong-Kwan campus of the NTUH, located in the Ta-An District, is among one of the field sites for the annual HEEP.
Most subjects who signed up for the annual HEEP in the NTUH are independent community-dwelling elders of Ta-An District of Taipei City. The following assessments are provided in a typical HEEP: questionnaires for chronic disease profile, physical examinations and anthropometric measures, chest $\mathrm{X}$ ray, electrocardiogram, urine/stool examination, and laboratory analyses including complete blood count, liver function, renal function, triglyceride, and cholesterol.

Participants of the current study were randomly selected from those who signed up for the HEEP in the NTUH. Participants of HEEP was excluded from the study if they had a known diagnosis of dementia; were functionally impaired requiring assistance in dressing, personal hygiene, or bathing; were terminally ill; were contra-indicated for MRI examination such as claustrophobia or pacemaker implantation; were unavailable during the study period; had impairment in vision, hearing, or communicative ability, hence making participation in the study impossible; or had histories of myocardial infarction or stroke.

From October 2006 through December 2007, 93 eligible individuals agreed to participate and had complete information in interview data, brain MRI, PWV measurement, and laboratory examinations. The NTUH Institutional Review Board reviewed and approved the study. Informed consents were obtained from all participants.

\subsection{Sociodemographic and interview data}

Age, gender, educational level, averaged hours of sleep per night, and status of smoking and alcohol drinking were obtained by self-report. Co-morbidities including hypertension, diabetes mellitus, heart disease (defined as a history of coronary heart disease, congestive heart failure, or angina), chronic lung disease, depressive disorder were ascertained by self-report questionnaires. During the interview, we asked participants to bring all medications, including vitamin supplements and over-the-counter medications, that they were actively taking. Cognitive function was ascertained using Mini-Mental State Examination. Center for Epidemiologic Studies Depression Scale (CES-D), ranged from 0 to 60 with a higher score indicating more depressive symptom, was used to ascertain depression symptom.

\subsection{Metabolic risks}

Two supine blood pressure (BP) determinations were taken by a trained research assistant with a mercury sphygmomanometer. BPs were measured in the right arm unless otherwise specified. Averaged systolic BP (SBP) and diastolic BP (DBP) were obtained. Pulse pressure (PP) was defined as averaged SBP minus averaged DBP. Waist circumference was measured at the iliac crest to the nearest $0.1 \mathrm{~cm}$. Blood specimens were processed in the NTUH central laboratories for analysis. Levels of serum total cholesterol and triglycerides were measured enzymatically, and levels of HDL cholesterol were measured using 
precipitation. Serum fasting glucose levels were processed by using the hexokinase enzymatical method. Use of antihypertensives, lipid-lowering agents, and anti-diabetic medications, including oral hypoglycemic agents or insulin injection, were ascertained by self-report questionnaires.

\subsection{Pulse wave velocity}

PWV was measured with the subject in a supine position after $15 \mathrm{~min}$ of rest using an automatic waveform analyzer (Colin VP-2000, Omeron Inc., Japan). The instrument simultaneously recorded right and left brachial and tibial arterial pressure wave forms, lead I of the electrocardiogram, and a phonocardiogram. A carotid tonometry sensor incorporating an array of 15 micropiezoresistive transducers was coupled with this device for analysis of common carotid arterial wave. Occlusion cuffs, connected to both plethysmographic and oscillometric sensors, were placed around both arms and ankles for pulse wave analysis and BP measurements. The time difference between the brachial and ankle arterial pressure wave was determined by the wave front velocity theory. The distance between the arm and ankle was automatically calculated based on the patient's height. Finally, the baPWV (brachial-ankle PWV) was calculated by dividing the distance with the time difference. Measurement of right and left baPWV was obtained for an average of $10 \mathrm{~s}$. The averaged left and right baPWVs of each subject were used for subsequent analyses. The haPWV (heart-ankle PWV) was derived by similar method. The time difference was the period between S2 heart sound and the initial point of tibial arterial pressure wave.

\subsection{MRI assessment}

Subjects underwent MR examination by a 1.5-T MR unit (Sigma, General Electric Healthcare System, Milwaukee, USA). The examination produced three sets of 5-mm-thick in axial, sagittal, and coronal lanes for localization. Then we obtained the standard axial images parallel to the AC and PC line. They included T1-weighted FLAIR images (TR/TE/ $\mathrm{TI}=2000 \mathrm{~ms} /$ minimal full $/ 720 \mathrm{~ms}$, matrix $=320 \times 192$, $\mathrm{FOV}=22 \mathrm{~cm}$, slice thickness $=3.5 \mathrm{~mm}$ ), axial proton density and T2-weighted images $(\mathrm{TR} / \mathrm{TE}=3425 \mathrm{~ms} / \mathrm{minimal}$ full and $103.7 \mathrm{~ms}$, matrix $=288 \times 192 . \mathrm{FOV}=22 \mathrm{~cm}$ ), and T2-weighted FLAIR images $(\mathrm{TR} / \mathrm{TE} / \mathrm{TI}=9000 \mathrm{~ms} / 80 \mathrm{~ms} / 2250 \mathrm{~ms}$, matrix $=288 \times 224, \mathrm{FOV}=22 \mathrm{~cm}$ ).

Imaging data, archived electronically, were sent to NTUH Department of Medical Imaging reading center for interpretation by a neuroradiologist (Liu HM) without knowledge of participants' clinical information. Fazekas rating scale was used to describe two different types of white matter hyperintense signal abnormalities, or WML, surrounding the ventricles and in the deep white matter [19]. Periventricular hyperintensity $(\mathrm{PVH})$ was graded as $0=$ absence, 1 (mild) $=$ "casps" or pencil-thin lining, 2 (moderate) $=$ smooth "halo", 3 (severe)=irregular PVH extending into the deep white matter. Separate deep white matter hyperintense signals $(\mathrm{DWMH})$ were rated as $0=$ absence, 1 (mild) $=$ punctuate foci, 2 (moderate) $=$ beginning confluence of foci, 3 (severe) $=$ large confluent areas. Total burden of WML, defined as WML grade, was estimated by summing the PVH grade and the DWMH grade, forming a scale from 0 to 6 . The presence of silence infarct, as defined as asymptomatic hyperintense

Table 1

Characteristics of study participants

\begin{tabular}{|c|c|c|c|c|}
\hline Characteristics & Women $(n=48)$ & Men $(n=45)$ & $P$ value & Total \\
\hline \multicolumn{5}{|l|}{ Continuous variables $^{a}$} \\
\hline Age (year) & $70.69(4.39)$ & $74.34(5.92)$ & 0.001 & $72.46(5.47)$ \\
\hline Education (year) & $12.42(2.66)$ & $13.89(2.78)$ & 0.011 & $13.13(2.80)$ \\
\hline Body mass index $(\mathrm{kg} / \mathrm{m} 2)$ & $23.93(3.17)$ & $23.80(2.80)$ & 0.836 & $23.87(2.98)$ \\
\hline Averaged hours of sleeping per night (h) & $5.48(0.99)$ & $6.46(0.97)$ & $<0.001$ & $5.95(1.09)$ \\
\hline MMSE score $(0-30)$ & $29.27(0.92)$ & $29.36(1.19)$ & 0.700 & $29.31(1.05)$ \\
\hline CES-D score $(0-60)^{\mathrm{c}}$ & $4.54(6.70)$ & $3.07(4.94)$ & 0.232 & $3.83(5.93)$ \\
\hline haPWV $(\mathrm{cm} / \mathrm{s})$ & $1090(118)$ & $1134(133)$ & 0.098 & $1112(127)$ \\
\hline $\mathrm{baPWV}(\mathrm{cm} / \mathrm{s})$ & $1687(276)$ & $1712(328)$ & 0.684 & $1699(301)$ \\
\hline \multicolumn{5}{|l|}{ Categorical variables ${ }^{\mathrm{b}}$} \\
\hline Current smoker & $0(0)$ & $3(6.67)$ & 0.069 & $3(3.23)$ \\
\hline Not drinking alcohol & $28(58.33)$ & $13(28.89)$ & 0.004 & $41(44.09)$ \\
\hline History of hypertension & $24(50)$ & $23(51.11)$ & 0.915 & $47(50.54)$ \\
\hline History of diabetes mellitus & $5(10.42)$ & $5(11.11)$ & 0.914 & $10(10.75)$ \\
\hline Heart disease & $8(16.67)$ & $6(13.33)$ & 0.653 & $14(15.05)$ \\
\hline Silent infarct & $1(2.08)$ & $4(8.89)$ & 0.146 & $5(5.38)$ \\
\hline Moderate-to-severe PVH & $3(6.25)$ & $12(26.67)$ & 0.007 & $15(16.13)$ \\
\hline Moderate-to-severe DWMH & $7(14.58)$ & $10(22.22)$ & 0.341 & $17(18.28)$ \\
\hline Moderate-to-severe PVH or DWMH & $7(14.58)$ & $15(33.33)$ & 0.033 & $22(23.66)$ \\
\hline
\end{tabular}

Abbreviations: baPWV, brachial-ankle pulse wave velocity; CES-D, Center for Epidemiologic Studies Depression Scale; haPWV, heart-ankle pulse wave velocity; MMSE, Mini-Mental State Examination; WML, white matter lesion.

${ }^{\text {a }}$ Values in the continuous variables were expressed as mean (standard deviation).

${ }^{\mathrm{b}}$ Values in the categorical variables were expressed as number (percent).

${ }^{c}$ A higher score in CES-D indicates more depressive symptoms. 
Table 2

Age and sex-adjusted association between potential risk factors for white matter hyperintensity and arterial stiffness ${ }^{\mathrm{a}}$

\begin{tabular}{|c|c|c|c|c|c|c|}
\hline \multirow[t]{2}{*}{ Potential risk factors } & \multicolumn{2}{|l|}{ WML grade } & \multicolumn{2}{|l|}{ baPWV } & \multicolumn{2}{|l|}{ haPWV } \\
\hline & $\beta(\mathrm{SE})$ & $p$ & $\beta(\mathrm{SE})$ & $p$ & $\beta(\mathrm{SE})$ & $p$ \\
\hline \multicolumn{7}{|l|}{ Demographics } \\
\hline Years of education (year) & $-0.055(0.05)$ & 0.273 & $-13.25(11.20)$ & 0.240 & $-1.16(4.50)$ & 0.798 \\
\hline Live alone & $0.542(0.523)$ & 0.303 & $159.74(117.31)$ & 0.177 & $62.62(46.79)$ & 0.184 \\
\hline Income $>60,000$ NTD per month & $-0.288(0.285)$ & 0.314 & $-50.96(64.39)$ & 0.431 & $-20.75(25.67)$ & 0.421 \\
\hline \multicolumn{7}{|l|}{ Disease/medication history } \\
\hline Hypertension & $0.714(0.256)$ & 0.007 & $101.57(59.25)$ & 0.090 & $53.05(23.34)$ & 0.025 \\
\hline Diabetes mellitus & $-0.292(0.43)$ & 0.499 & $191.25(95.08)$ & 0.047 & $76.62(37.90)$ & 0.046 \\
\hline Heart diseases & $-0.17(0.38)$ & 0.656 & $-118.57(84.9)$ & 0.166 & $-42.41(33.92)$ & 0.214 \\
\hline Chronic lung disease & $-0.479(0.429)$ & 0.267 & $10.33(97.38)$ & 0.916 & $-18.02(38.78)$ & 0.643 \\
\hline Depressive disorder & $-1.154(0.746)$ & 0.125 & $-47.89(170.41)$ & 0.779 & $-56.47(67.71)$ & 0.407 \\
\hline Use of anti-hypertensive & $0.708(0.257)$ & 0.007 & $78.49(59.69)$ & 0.192 & $34.31(23.75)$ & 0.152 \\
\hline Use of lipid-lowering agents & $0.012(0.452)$ & 0.980 & $51.34(101.71)$ & 0.615 & $25.69(40.52)$ & 0.528 \\
\hline \multicolumn{7}{|l|}{ Health-related behaviors } \\
\hline Current smoker & $0.401(0.794)$ & 0.615 & $-136.6(178.68)$ & 0.447 & $-11.22(71.47)$ & 0.876 \\
\hline Not drinking alcohol at all & $0.164(0.281)$ & 0.561 & $-62.86(63.17)$ & 0.322 & $-19.20(25.25)$ & 0.449 \\
\hline Averaged hours of sleep per night (hour) & $0.184(0.139)$ & 0.187 & $1.14(31.56)$ & 0.971 & $6.37(12.57)$ & 0.613 \\
\hline \multicolumn{7}{|l|}{ Metabolic risks } \\
\hline Systolic blood pressure (mmHg) & $0.016(0.008)$ & 0.035 & $6.83(1.62)$ & $<0.001$ & $3.16(0.62)$ & $<0.001$ \\
\hline Diastolic blood pressure (mmHg) & $0.028(0.011)$ & 0.017 & $3.23(2.63)$ & 0.224 & $1.63(1.04)$ & 0.122 \\
\hline Pulse pressure (mmHg) & $0.005(0.009)$ & 0.574 & $6.51(1.82)$ & 0.001 & $2.94(0.71)$ & $<0.001$ \\
\hline Fasting glucose (mg/dL) & $-0.013(0.009)$ & 0.161 & $2.27(2.02)$ & 0.265 & $1.07(0.80)$ & 0.188 \\
\hline Waist circumference $(\mathrm{cm})$ & $0.012(0.017)$ & 0.474 & $4.68(3.84)$ & 0.226 & $0.81(1.54)$ & 0.600 \\
\hline Triglyceride (mg/dL) & $0.003(0.002)$ & 0.185 & $1.55(0.52)$ & 0.004 & $0.79(0.20)$ & $<0.001$ \\
\hline HDL cholesterol (mg/dL) & $-0.001(0.012)$ & 0.965 & $-6.07(2.65)$ & 0.024 & $-1.71(1.07)$ & 0.114 \\
\hline Total cholesterol (mg/dL) & $0.006(0.004)$ & 0.176 & $0.54(0.99)$ & 0.589 & $0.39(0.39)$ & 0.327 \\
\hline Metabolic $Z$ score & $0.051(0.049)$ & 0.298 & $39.15(10.20)$ & $<0.001$ & $15.86(4.06)$ & $<0.001$ \\
\hline
\end{tabular}

Abbreviations: baPWV, brachial-ankle pulse wave velocity; haPWV, heart-ankle pulse wave velocity; WML, white matter lesion.

a Separate multiple linear regression was performed with WMH grade, baPWV, and haPWV as dependent variables and with age, sex, and each potential risk factor as independent variables. Parameter estimates $(\beta)$ can be interpreted as differences in WMH grade, haPWV, and haPWV with 1 -unit change in the potential risk factor.

on T2-weighted images while hypointense on T1-weighted images, was also recorded.

\subsection{Statistical analysis}

Differences in baseline characteristics between men and women were compared by Student's $t$-test, Wilcoxon Rank Sum test, or chi-squared test. To examine the association of potential risk factors with WML and PWV, we performed multiple linear regression, with WML and PWV as the dependent variables and with age, sex, and the potential risk factors as independent variables. To consider various metabolic risk factors as a whole, we also calculated the metabolic $Z$ score. The score was derived by converting each component of the metabolic risk factors, namely blood pressure, waist circumference, fasting glucose level, serum triglyceride level, and serum HDL level, into a $Z$ score based on means of the study population.[20] The metabolic $Z$ score was calculated by summation of the former four $Z$ scores minus the HDL $Z$ score. The relations of metabolic risk, by utilizing the composite metabolic $Z$ score, to PWV and WML were assessed by using multiple linear regression.

The associations of baPWV and haPWV (dependent variables) with WML (independent variables), including PVH, DWMH, and WML grade, respectively, were then examined using multiple linear regression while adjusting for age, sex, metabolic $Z$ score, presence of silence infarct, and the use of anti-hypertensives, lipid lowering agents, and anti-diabetic agents. Participants without any hyperintense abnormalities, i.e. those who had grade $0 \mathrm{PVH}$, grade 0 $\mathrm{DWMH}$, or grade $0 \mathrm{WMH}$, were regarded as the reference groups respectively. Adjusted means of baPWV and haPWV

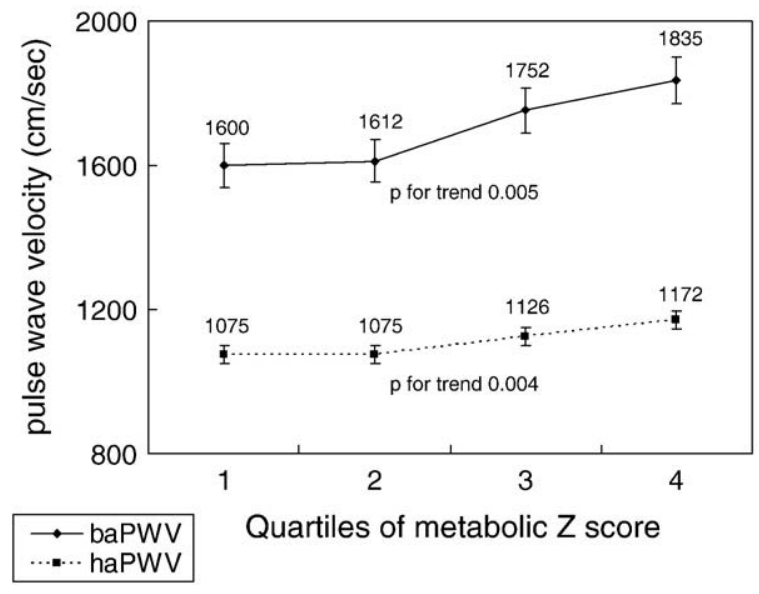

Fig. 1. Adjusted means of pulse wave velocity across quartiles of metabolic $Z$ score. 
in different categories of white matter hyperintense abnormalities were obtained by multiple linear regression.

We estimated potential cutoff values of baPWV and haPWV to predict the presence of moderate to severe white matter hyperintense abnormalities, namely, grades 2 to 3 $\mathrm{PVH}$ and grades 2 to $3 \mathrm{DWMH}$, via the receiver operating characteristics (ROC) analyses. The ROC analyses gave an estimate of the overall discriminative ability of PWV by the area under the curve (AUC) statistic. We calculated sensitivity and specificity across the range of possible cutoff PWV values. The ROC curve was plotted with sensitivity on the $y$-axis and (1-specificity) on the $x$-axis. AUC and associated $95 \%$ confidence intervals (CIs) were calculated.

\section{Results}

\subsection{Characteristics of study population}

A total of 93 participants successfully completed the assessments of MRI and PWV, as well as clinical and laboratory examinations. Selected baseline characteristics of the study population as a whole $(N=93$, mean age 72.46 years) and by gender were summarized in Table 1. Forty-eight female participants represented $52 \%$ of the study population. Eighty participants $(86 \%)$ had various degree of WML. Nearly half (47\%) of the study participants had hypertension, $10 \%$ diabetes mellitus, and 14\% heart diseases. Men tended to be older, to have higher education, greater hand grip strength, higher grades of $\mathrm{PVH}$, and more hours of night sleep than women did. There was no difference in terms of chronic co-morbidities (hypertension, diabetes mellitus, heart disease), PWV, BMI, MMSE scores, depressive symptom, and habitual gait speed between men and women.

\subsection{Potential correlates of arterial stiffness and WML}

After adjusting for age and sex, WML grade was significantly associated with a history of hypertension, systolic and diastolic blood pressures, and use of anti-hypertensives, but not with educational level, living status (whether to live alone or to live with family members), income, smoking status, alcohol drinking, averaged hours of sleep per night, or the presence of diabetes mellitus, heart diseases, chronic lung disease, or depressive disorder (Table 2).

Age-, and sex-adjusted regression analysis showed that PWV was related to the presence of diabetes mellitus and several metabolic risks. Both baPWV and haPWV were positively associated with systolic blood pressure, pulse pressure, and triglyceride. Additionally, baPWV was significantly associated with HDL cholesterol $(\beta=-6.07$, $p=0.024)$ while haPWV with the presence of hypertension $(\beta=53.05, p=0.025)$. The metabolic $Z$ score, representing the metabolic risks as a whole, was positively associated with both baPWV and haPWV ( $p<0.001$, respectively). The adjusted means of baPWV and haPWV (controlling for age, sex, smoking status, alcohol drinking, use of anti-hypertensives and lipid-lowering agents) based on different metabolic $Z$ score quartiles were illustrated in Fig. 1. The trends for the relations of metabolic $Z$ score quartiles to both baPWV and haPWV were statistically significant $(p=0.005$ and 0.004 , respectively).

\subsection{Association between WML and arterial stiffness}

PWV was associated with white matter hyperintensity. After controlling for age, sex, metabolic $Z$ score, presence of silent infarct, and use of anti-hypertensives, lipid-lowering, and anti-diabetic agents, participants in the highest grade of PVH had significantly higher baPWV compared to those without PVH $(\beta$ coefficient $=357.42, p$ value $=0.035)$ (Table 3). Moreover, participants with the highest grade of DWMH tended to have higher baPWV and haPWV compared to those without DWMH ( $\beta$ coefficient for baPWV $=385.24, \quad p$ value $=0.018 ; \beta$ coefficient for haPWV $=183.82, p$ value $=0.005$ ). Total burden of WML, i.e. WML grade, was also correlated with baPWV and haPWV. Participant who had grades 5 to $6 \mathrm{WML}$ had higher baPWV and haPWV compared to those without any WML $(\beta$ coefficient for baPWV $=366.68, p$ value $=0.035 ; \beta$ coefficient for haPWV $=171.81, p$ value $=0.012$ ). Adjusted means of baPWV and haPWV in different grades of white matter signal abnormalities were illustrated in Fig. 2.

\subsection{ROC curves, sensitivity, and specificity}

Fig. 3 displayed the ROC curves for the discriminative ability of haPWV and baPWV to identify the presence of

Table 3

Multivariate-adjusted regression analyses for the associations between white matter hyperintense signal abnormalities and pulse wave velocity (PWV)

\begin{tabular}{|c|c|c|c|c|}
\hline \multirow[t]{2}{*}{ MRI finding } & \multicolumn{2}{|l|}{ baPWV } & \multicolumn{2}{|l|}{ haPWV } \\
\hline & $\beta(\mathrm{SE})$ & $P$ value & $\beta(\mathrm{SE})$ & $P$ value \\
\hline \multicolumn{5}{|c|}{ Periventricular hyperintensities (PVH) } \\
\hline Grade $=0$ (reference) & Reference & & Reference & \\
\hline Grade $=1$ & $-12.24(74.46)$ & 0.870 & $-19.62(29.63)$ & 0.510 \\
\hline Grade $=2$ & $124.32(116.97)$ & 0.291 & $66.73(46.56)$ & 0.156 \\
\hline Grade $=3$ & $357.42(166.14)$ & 0.035 & $117.99(66.12)$ & 0.079 \\
\hline \multicolumn{5}{|c|}{ Deep white matter hyperintensities (DWMH) } \\
\hline Grade $=0$ (reference) & Reference & & Reference & \\
\hline Grade $=1$ & $-63.27(65.41)$ & 0.341 & $-21.29(26.09)$ & 0.417 \\
\hline Grade $=2$ & $8.12(97.85)$ & 0.935 & $17.05(38.98)$ & 0.663 \\
\hline Grade $=3$ & $385.24(154.47)$ & 0.018 & $183.82(62.81)$ & 0.005 \\
\hline \multicolumn{5}{|c|}{ White matter lesion (WML) } \\
\hline Grade $=0$ (reference) & Reference & & Reference & \\
\hline Grade $=1-2$ & $-74.17(89.36)$ & 0.409 & $-36.24(34.94)$ & 0.303 \\
\hline Grade $=3-4$ & $8.70(107.60)$ & 0.936 & $19.13(42.07)$ & 0.651 \\
\hline Grade $=5-6$ & $366.68(170.20)$ & 0.035 & $171.81(66.55)$ & 0.012 \\
\hline
\end{tabular}

Separate multiple linear regression was performed with baPWV and haPWV as dependent variables and with white matter hyperintense signal abnormalities as independent variable while adjusted for age, sex, metabolic $Z$ score silent infarct, and use of anti-hypertensives, lipid-lowering, and anti-diabetic agents. 

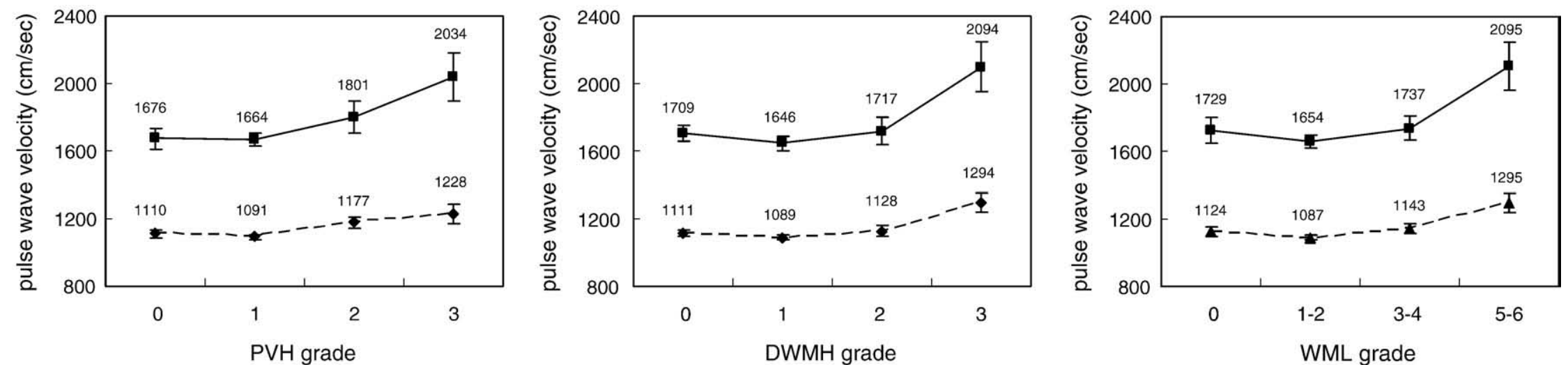

Fig. 2. Adjusted means of pulse wave velocity in different grades of white matter hyperintense abnormalities. Solid line refers to brachial-ankle pulse wave velocity. Dashed line refers to heart-ankle pulse wave velocity. The adjusted covariates include age, sex, metabolic $Z$ score, presence of silent infarct, and use of anti-hypertensives, lipid-lowering, and anti-diabetic agents. 
moderate-to severe PVH and DWMH. We demonstrated that, compared to baPWV, haPWV was a better measurement in identifying the presence of moderate-to-severe WMLs. AUCs for haPWV and baPWV to identify moderate-tosevere $\mathrm{PVH}$ were 0.78 (95\% confidence interval $[\mathrm{CI}]=0.66-$ $0.91)$ and $0.68(95 \% \mathrm{CI}=0.54-0.82)$, respectively. AUCs for haPWV and baPWV to identify moderate-to-severe DWMH were $0.72(95 \% \mathrm{CI}=0.59-0.85)$ and $0.66(95 \% \mathrm{CI}=0.53-$ $0.80)$, respectively.

The optimal cutoffs to identify moderate-to-severe PVH and DWMH were determined by calibrating the haPWV values to find a best balance between sensitivity and specificity. A range of possible cutoff values were provided in the Table 4. Using a haPWV cutoff of $1177 \mathrm{~cm} / \mathrm{sec}$, the sensitivity and specificity in identifying grades $2-3 \mathrm{PVH}$

(a)

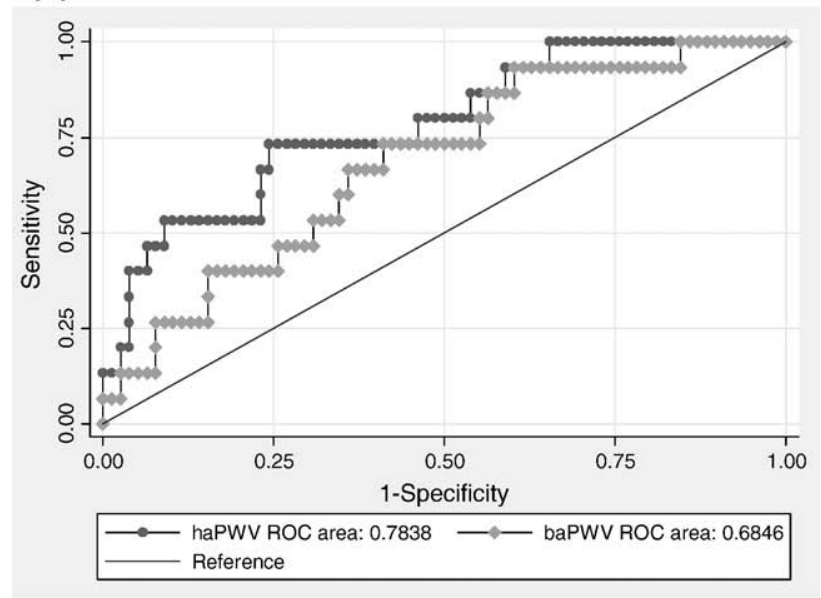

(b)

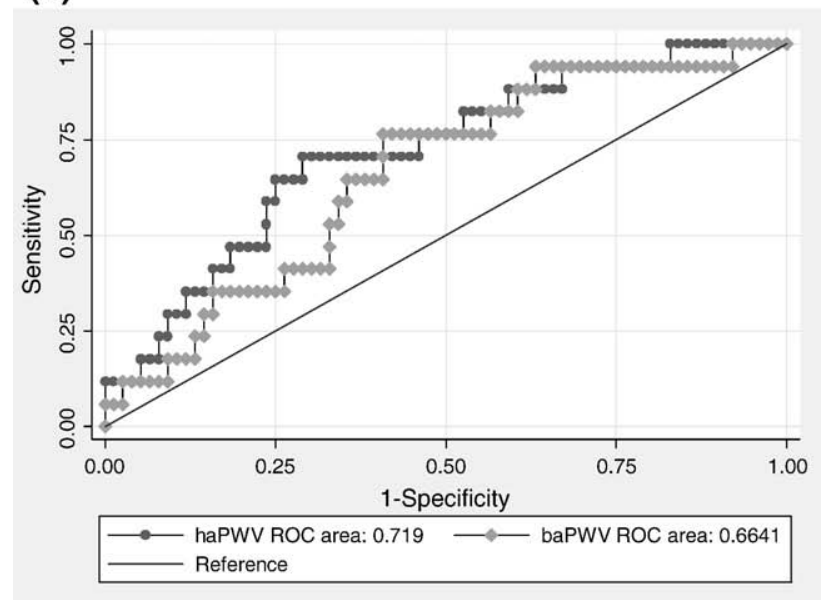

Fig. 3. Receiver operating characteristic (ROC) curves for haPWV and baPWV to identify the presence of moderate-to-severe white matter hyperintense abnormalities, including PVH (Fig. 2-a) and DWMH (Fig. 2-b). AUCs for haPWV and baPWV to identify moderate-to-severe PVH were $0.78(95 \%$ confidence interval $=0.66-0.91)$ and $0.68(95 \%$ confidence interval $=0.54$ $0.82)$, respectively. AUCs for haPWV and baPWV to identify moderate-tosevere DWMH were $0.72(95 \%$ confidence interval $=0.59-0.85)$ and 0.66 $(95 \%$ confidence interval $=0.53-0.80)$, respectively.
Table 4

Sensitivity and specificity of pulse wave velocity in identifying moderate-tosevere white matter hyperintense abnormalities

\begin{tabular}{|c|c|c|c|c|}
\hline \multirow{2}{*}{$\begin{array}{l}\text { haPWV cutoff } \\
(\mathrm{cm} / \mathrm{sec})\end{array}$} & \multicolumn{2}{|c|}{ PVH ( $\geq$ grade 2 ) } & \multicolumn{2}{|c|}{ DWMH $(\geq$ grade 2$)$} \\
\hline & Sensitivity & Specificity & Sensitivity & Specificity \\
\hline 1043 & 0.93 & 0.40 & 0.88 & 0.39 \\
\hline 1053 & 0.87 & 0.41 & 0.82 & 0.41 \\
\hline 1071 & 0.80 & 0.46 & 0.82 & 0.47 \\
\hline 1085 & 0.80 & 0.50 & 0.76 & 0.50 \\
\hline 1092 & 0.73 & 0.54 & 0.71 & 0.54 \\
\hline 1160 & 0.73 & 0.71 & 0.71 & 0.71 \\
\hline 1177 & 0.73 & 0.76 & 0.65 & 0.75 \\
\hline 1190 & 0.60 & 0.77 & 0.53 & 0.76 \\
\hline 1195 & 0.53 & 0.79 & 0.47 & 0.79 \\
\hline 1208 & 0.53 & 0.82 & 0.47 & 0.82 \\
\hline 1253 & 0.47 & 0.91 & 0.29 & 0.88 \\
\hline 1273 & 0.40 & 0.94 & 0.24 & 0.91 \\
\hline
\end{tabular}

were 0.73 and 0.76 , respectively. The sensitivity and specificity in identifying DWMH were both 0.71 if the haPWV cutoff was $1160 \mathrm{~cm} / \mathrm{s}$.

\section{Discussion}

Our study demonstrated that arterial stiffness was a risk factor for WML independent of age, sex, metabolic composite score including blood pressure, waist circumference, fasting glucose level, serum triglyceride level, and serum HDL level, as well as the use of anti-hypertensives, lipid-lowering, and anti-diabetic agents. Our findings supported and extended previous studies examining the relationship between cerebral small vessel disease and arterial stiffness. Ohmine and colleagues investigated the association between arterial stiffness and MR white matter hyperintensities in 132 community-dwelling older Japanese and showed that baPWV was independently associated with the appearance of WML on MRI, in particular PVH [21]. In patients of hypertension, researchers demonstrated that increased arterial stiffness was associated with silent cerebral small-vessel disease shown in MRI [22,23]. We consolidated these findings by further demonstrating that PWV was a fairly good marker in identifying the presence of radiographically evident WML. The AUCs, representing the discriminative power, for haPWV to identify the presence of moderate-tosevere PVH and DWMH were 0.78 and 0.72 , respectively.

Our study has the following implication. PWV has recently attracted significant research interest in that measurements of PWV have been found useful in assessing cardiovascular risk. Compelling evidence has supported the notion that increased arterial stiffness is a risk factor for cardiovascular events [24-26]. In fact, the associations between arterial stiffness and adverse cardiovascular outcomes have been documented in a wide range of populations, including hypertensive individuals [24], persons with end-stage renal disease [27], as well as general population $[25,26]$. In addition to being a predictor of adverse 
cardiovascular outcomes, PWV, a simple and noninvasive measurement of arterial stiffness using an automatic device, appears to be a significant indicator for preclinical cerebrovascular disease as well. Even after adjusting for the composite metabolic risk score, PWV, significantly refining the risk stratification above and beyond classic metabolic risk factors, still remains its significant association with WML. Taken together, measurement of PWV could be considered as a useful tool in identifying susceptible individuals for the development of potential sequelae of WML, namely cognitive impairment, gait/balance problems, or falls - collectively called geriatric syndromes. Identification of PWV as an important correlate of WML can thus facilitate appropriate interventions, including lifestyle alterations, pharmaceutical management, or physical rehabilitation, in persons with such preclinical, asymptomatic endophenotypes.

Our study has potential limitations that deserve comment. Because of the cross-sectional design, causal relationship between arterial stiffness and WML cannot be established. Atherosclerotic change of cerebrovascular circulation is generally considered to have a crucial role in the pathogenesis of WML. The relationship between arterial stiffness and WML should be explored prospectively. Second, although we have demonstrated the optimal cutoff values of haPWV to identify moderate-to-severe PVH and DWMH, the sensitivity and specificity were not high. Therefore, utilizing PWV as a surrogate marker for high grade WML needs to be further studied. Lastly, our study has weakness in terms of generalizability because the study population was composed of high-functioning older adults who voluntarily participated the health evaluation. The participants were free of dementia and physical limitation, with a mean MMSE score of 29.3 and none of the participants requiring assistance in activities of daily living. Although we provided important insights regarding potential preventive strategies in totally asymptomatic, otherwise healthy older adults, the association between arterial stiffness and WML among general population needs further investigation.

In conclusion, greater PWV is associated with higher grades of WML among high-functioning older adults. Efforts to prevent arterial stiffening through reduction of cardiovascular risks will likely ameliorate the progression of WML, thus improving the health outcomes of older adults. Our findings support the notion that measurement of arterial stiffness is useful in clinical practice for risk stratification.

\section{Acknowledgement}

The study was sponsored by the National Health and Research Institutes (Project code: GE-095-CP0) and the Department of Industrial Technology, Ministry of Economic Affairs, Taiwan (Project code 95-EC-17-A-19-S1-016).

The authors of this manuscript have certified that they comply with the Principles of Ethical Publishing in the International Journal of Cardiology [28].

\section{References}

[1] Manolio TA, Kronmal RA, Burke GL, et al. Magnetic resonance abnormalities and cardiovascular disease in older adults. The Cardiovascular Health Study. Stroke 1994;25(2):318-27.

[2] de Leeuw FE, de Groot JC, Achten E, et al. Prevalence of cerebral white matter lesions in elderly people: a population based magnetic resonance imaging study. The Rotterdam Scan Study. J Neurol Neurosurg Psychiatry 2001;70(1):9-14.

[3] Kuo HK, Lipsitz LA. Cerebral white matter changes and geriatric syndromes: is there a link? J Gerontol A Biol Sci Med Sci 2004;59 (8):818-26.

[4] Marstrand JR, Garde E, Rostrup E, et al. Cerebral perfusion and cerebrovascular reactivity are reduced in white matter hyperintensities. Stroke 2002;33(4):972-6.

[5] Fazekas F, Kleinert R, Offenbacher H, et al. Pathologic correlates of incidental MRI white matter signal hyperintensities. Neurology 1993;43(9):1683-9.

[6] Bots ML, van Swieten JC, Breteler MM, et al. Cerebral white matter lesions and atherosclerosis in the Rotterdam Study. Lancet 1993;341 (8855):1232-7.

[7] Dufouil C, de Kersaint-Gilly A, Besancon V, et al. Longitudinal study of blood pressure and white matter hyperintensities: the EVA MRI Cohort. Neurology 2001;56(7):921-6.

[8] de Leeuw FE, de Groot JC, Oudkerk M, et al. Hypertension and cerebral white matter lesions in a prospective cohort study. Brain 2002;125(Pt 4):765-72.

[9] Taylor WD, MacFall JR, Provenzale JM, et al. Serial MR imaging of volumes of hyperintense white matter lesions in elderly patients: correlation with vascular risk factors. AJR Am J Roentgenol 2003;181 (2):571-6.

[10] Matsubayashi K, Okumiya K, Wada T, et al. Postural dysregulation in systolic blood pressure is associated with worsened scoring on neurobehavioral function tests and leukoaraiosis in the older elderly living in a community. Stroke 1997;28(11):2169-73.

[11] Puisieux F, Monaca P, Deplanque D, et al. Relationship between leukoaraiosis and blood pressure variability in the elderly. Eur Neurol 2001;46(3):115-20.

[12] Kalimo H, Viitanen M, Amberla K, et al. hereditary disease of arteries causing brain infarcts and dementia. Neuropathol Appl Neurobiol 1999;25(4):257-65.

[13] Hassan A, Lansbury A, Catto AJ, et al. Angiotensin converting enzyme insertion/deletion genotype is associated with leukoaraiosis in lacunar syndromes. J Neurol Neurosurg Psychiatry 2002;72(3):343-6.

[14] Laurent S, Boutouyrie P. Recent advances in arterial stiffness and wave reflection in human hypertension. Hypertension 2007;49(6):1202-6.

[15] Cockcroft JR, Webb DJ, Wilkinson IB. Arterial stiffness, hypertension and diabetes mellitus. J Hum Hypertens 2000;14(6):377-80.

[16] Tedesco MA, Natale F, Di Salvo G, Caputo S, Capasso M, Calabro R. Effects of coexisting hypertension and type II diabetes mellitus on arterial stiffness. J Hum Hypertens 2004;18(7):469-73.

[17] Blacher J, Guerin AP, Pannier B, Marchais SJ, Safar ME, London GM Impact of aortic stiffness on survival in end-stage renal disease. Circulation 1999;99(18):2434-9.

[18] Mattace-Raso FU, van der Cammen TJ, Hofman A, et al. Arterial stiffness and risk of coronary heart disease and stroke: the Rotterdam Study. Circulation 2006;113(5):657-63.

[19] Fazekas F, Chawluk JB, Alavi A, Hurtig HI, Zimmerman RA. MR signal abnormalities at $1.5 \mathrm{~T}$ in Alzheimer's dementia and normal aging. AJR Am J Roentgenol 1987;149(2):351-6.

[20] Lin LY, Kuo HK, Lai LP, Lin JL, Tseng CD, Hwang JJ. Inverse correlation between heart rate recovery and metabolic risks in healthy children and adolescents: insight from the National Health and Nutrition Examination Survey 1999-2002. Diabetes Care 2008;31(5):1015-20.

[21] Ohmine T, Miwa Y, Yao H, et al. Association between arterial stiffness and cerebral white matter lesions in community-dwelling elderly subjects. Hypertens Res 2008;31(1):75-81. 
[22] Henskens LH, Kroon AA, van Oostenbrugge RJ, et al. Increased aortic pulse wave velocity is associated with silent cerebral small-vessel disease in hypertensive patients. Hypertension 2008;52(6):1120-6.

[23] Kim DH, Kim J, Kim JM, Lee AY. Increased brachial-ankle pulse wave velocity is independently associated with risk of cerebral ischemic small vessel disease in elderly hypertensive patients. Clin Neurol Neurosurg 2008;110(6):599-604.

[24] Laurent S, Boutouyrie P, Asmar R, et al. Aortic stiffness is an independent predictor of all-cause and cardiovascular mortality in hypertensive patients. Hypertension 2001;37(5):1236-41.

[25] Sutton-Tyrrell K, Najjar SS, Boudreau RM, et al. Elevated aortic pulse wave velocity, a marker of arterial stiffness, predicts cardiovascular events in well-functioning older adults. Circulation 2005;111(25): 3384-90.

[26] Willum-Hansen T, Staessen JA, Torp-Pedersen C, et al. Prognostic value of aortic pulse wave velocity as index of arterial stiffness in the general population. Circulation 2006;113(5):664-70.

[27] Blacher J, Pannier B, Guerin AP, Marchais SJ, Safar ME, London GM. Carotid arterial stiffness as a predictor of cardiovascular and all-cause mortality in end-stage renal disease. Hypertension 1998;32(3):570-4.

[28] Coats AJ. Ethical authorship and publishing. Int J Cardiol 2009;131: 149-50. 